\title{
Misdiagnosis of bipolar disorder in patients with brain metastasis affecting frontal lobes
}

\author{
Bernardo Dell'Osso, ${ }^{1,2,3^{*}}$ (1) and Alberto Priori ${ }^{3,4}$
}

\author{
${ }^{1}$ Department of Biomedical and Clinical Sciences, University of Milan, Psychiatric Unit 2, ASST Fatebenefratelli-Sacco, Milan, Italy \\ ${ }^{2}$ Department of Psychiatry and Behavioral Sciences, Bipolar Disorders Clinic, Stanford Medical School, Stanford University, Stanford, CA, \\ USA \\ ${ }^{3}$ CRC "Aldo Ravelli" for Neurotechnology and Experimental Brain Therapeutics, University of Milan, Milan, Italy \\ ${ }^{4}$ Department of Health Sciences, University of Milan \& ASST Santi Paolo e Carlo, Milan, Italy
}

Received 17 December 2018; Accepted 18 December 2018

We read with the utmost interest the Letter to the Editor by Dr Gama Marques, ${ }^{1}$ which was in response to the publication of our review article "Psychiatric, behavioral, and cognitive disorders in patients with extracranial cancers." ${ }^{2}$ The Letter describes two cases of patients with behavioral alterations and undiagnosed brain metastases, who were acutely admitted as bipolar patients with current manic episodes in a psychiatric inpatient unit. Patients were subsequently diagnosed through neuroimaging as affected by brain metastasis and, ultimately, they were transferred to different, more appropriate, medical units with fatal outcome.

After stressing how frequent psychiatric manifestations of cranial and extracranial cancers may be in clinical practice, and after pointing out some useful epidemiologic statements to be kept in consideration by clinicians dealing with patients like those described in his report, Dr Gama Marques concludes with two provocative questions: why current recommended diagnostic pathways for first episode psychosis are not properly adopted, and why brain tumors can go undiagnosed despite available imaging facilities. In an attempt to reply to Dr Gama Marques' questions, we herein provide the following considerations.

What struck us about the first case, besides the lack of imaging investigation, was that the patient had been already diagnosed with breast cancer - she was undergoing relapse prevention therapy - and that no history of Bipolar Disorder (BD) was reported. These two elements would actually make more unlikely a de novo

*Address correspondence to: Bernardo Dell'Osso, Department of Biomedical and Clinical Sciences, University of Milan, Psychiatric Unit 2, ASST Fatebenefratelli-Sacco, Milan, Italy.

(Email: bernardo.dellosso@unimi.it) diagnosis of BD in a 70-year-old woman and should have represented an indication for priority imaging investigation. On the other hand, the second case could have posed more problems in terms of differential diagnosis, as the patient was a 40 -year-old woman with no history of cancer but previous diagnosis of personality disorder and drug abuse. In this case, the age of the patient, her psychiatric history and the contemporary presence of depressed mood, mood congruent psychotic symptoms, and suicidal ideation made more plausible a longitudinal diagnosis of bipolar depression in $\mathrm{BD}$, type $\mathrm{I}$. Nonetheless, the presence of ataxia could represent the only unusual symptom of BD among the other reported ones and this should probably have recommended further imaging investigation.

While we agree with Dr Gama Marques about the nonsensical lack of imaging investigation in patients whose symptoms may not necessarily (or not exclusively) depend on psychiatric disorders, it is known that sometimes clinicians may be limited in their requests of additional, more expensive imaging procedures by restrictive economic policies of their institutions. Indeed, such a scenario does not only involve nonpsychiatrist doctors but, in turn, psychiatrists, whose patients can at a certain point manifest symptoms and behavioral alterations caused by medical illnesses including cancer, dementia, and other neurological disorders. In our experience, we had cases of patients referred to our clinic by general practitioners for major depression, who turned out to be affected by frontotemporal dementia, ${ }^{3}$ and in these cases genetic testing of progranulin gene mutation and C9ORF72 hexanucleotide repeat expansion, along with imaging investigation, confirmed the neurological diagnosis. Along with the points raised by Dr Gama 
Marques, we believe the presence of pervasive and persistent cognitive impairment along with other behavioral alterations manifested de novo, in the absence of positive family history for psychiatric disorders and major live-events, should always represent a red flag for implementing the routine approach to patients with or without psychiatric disorders with additional investigation in terms of neuroimaging and genetic testing. Psychiatric disorders are never mutually exclusive with other conditions of the central nervous system.

\section{REFERENCES:}

1. Gama Marques J. Brain metastasis affecting frontal lobe misdiagnosed as bipolar disorder, without any previously neuroimaging exam: Two case reports with fatal outcome. CNS Spectr. In press.

2. Pravettoni G, Dell'Osso B, Bocci T, et al. Psychiatric, behavioral, and cognitive disorders in patients with extracranial cancers. CNS Spectr. 2018; 23(6): 388-401.

3. Galimberti D,Dell'Osso B, Altamura AC, Scarpini E. Psychiatric symptoms in frontotemporal dementia: Epidemiology, phenotypes, and differential diagnosis. Biol Psychiatry. 2015; 78(10): 684-692. 